\title{
Academic Cheating among Adolescents in relation to Socio-Economic Status
}

\author{
Prof. Ashok K. Kalia ${ }^{1}$, Mr. Vinod Kumar
}

\section{ABSTRACT:}

Academic cheating is a phenomenon present at all levels of education. The present study has tried to explore the academic cheating among adolescents with different levels of socioeconomic status. A representative sample of 300 (150 male and 150 female) adolescents from urban and rural schools of five districts in Haryana was randomly selected. Academic Cheating Scale by Kalia \& Kirandeep (2011) and Socio-economic Status Scale by Kalia \& Sahu (2012) were used to assess academic cheating and socio-economic status of adolescents. ANNOVA followed by t-test was applied to study significant difference in academic cheating among adolescents with different levels of socio-economic status. The study revealed that adolescents having High socio-economic status were found to be significantly higher on academic cheating in comparison with adolescents having Low socio-economic. Similar results were observed for Male, Urban and Rural adolescents for same groups' comparison. No significant difference was found among female adolescents having high socio-economic status, middle socio-economic status and low socio-economic status. However no significant difference was observed for rest of groups' comparison.

Keywords: Academic Cheating, Socio Economic Status and Adolescents.

Academic cheating is a phenomenon present at all levels of education. The frequency of cheating in today's classrooms weakens educators 'efforts and threatens students' learning. Academic cheating is defined as fraud, deceit or dishonesty in an examination or in an assignment or in the class by using or attempting to use methods which are prohibited or inappropriate (Maslach. 2004). Variety of fraudulent acts and actions which fall under the umbrella term of academic cheating are lying or forging documents, buying papers, plagiarism, purposely not following the rules, altering results, furnishing false information regarding assignments, making up sources, creating interference in class during instruction, capitalizing on the weakness of persons, procedures, or processes to gain advantage. (Arent,1991; Moore, 1998; Packer, 1990; Pratt and Mclaghlin, 1989; Maslach, 2004; Cizek , 2003), Active cheating to improve one's own grade and

${ }^{1}$ Professor, Department of Education, MDU Rohtak, Haryana

${ }^{2}$ Research Scholar, Department of Education, MDU Rohtak, Haryana

(C) 2015 I A Kalia, V Kumar; licensee IJIP. This is an Open Access Research distributed under the terms of the Creative Commons Attribution License (http://creativecommons.org/licenses/by/2.0), which permits unrestricted use, distribution, and reproduction in any Medium, provided the original work is properly cited. 


\section{Academic Cheating among Adolescents in relation to Socio-Economic Status}

Passive cheating to assist others in improving their grades, Kalia (2005) and Cheating in Examination, Plagiarism, Creating an Improper disadvantage to other students, Lying about Academic Assignments, Interference during instructions, Damaging intellectual property. Kalia and Kirandeep (2011).

Literatures have found that Socio-economic background of parents influences to a great extent the academic performance of their children. (Devi and Mayuri, 2003; Panday and Maikhuri, 2003; Panigrahi, 2005; Alam, 2009; Mohanty, 2009). Socio Economic Status is the position that an individual or family occupies with reference to prevailing average standards of cultural possessions, effective income, material possessions, level of education and aspiration and participation in group activity of community Kalia and Sahu (2012). Kyglo (2001) was of opinion that students ${ }^{\text {ee }}$ behaviors to cheating in examination might be traceable to the influence of parents or home. Nzoka (2007) found children from high socio-economic status to exhibit cheating behaviour less than those from lower socio-economic status, their reasons being that, children from high socio-economic status have access to educational facilities/ materials among others. Aduloju and Obinne (2013) found that parent socio-economic status had no significant effect on students cheating behavior. Okorodudu (2013) also examined peer pressure and socioeconomic status as predictors of student's attitude to examination malpractice in Nigeria. Kalia and Kirandeep (2011) bear similar views that high socioeconomic status adolescents are significantly higher on academic cheating. The present research aims to study academic cheating among adolescents in relation to their socioeconomic status. This could be beneficial in looking at ways to prevent cheating behavior while children are at a young age.

\section{OBJECTIVES}

1. To study Academic Cheating among adolescents having High Socio Economic Status, Middle Socio Economic Status and Low Socio Economic Status.

2. To study Academic Cheating among Male adolescents having High Socio Economic Status, Middle Socio Economic Status and Low Socio Economic Status.

3. To study Academic Cheating among Female adolescents having High Socio Economic Status, Middle Socio Economic Status and Low Socio Economic Status.

4. To study Academic Cheating among Urban adolescents having High Socio Economic Status, Middle Socio Economic Status and Low Socio Economic Status.

5. To study Academic Cheating among Rural adolescents having High Socio Economic Status, Middle Socio Economic Status and Low Socio Economic Status.

\section{HYPOTHESIS}

1) There is no significant difference in academic cheating among adolescents having High Socio Economic Status, Middle Socio Economic Status and Low Socio Economic.

2) There is no significant difference in academic cheating among Male adolescents having High Socio Economic Status, Middle Socio Economic Status and Low Socio Economic.

3) There is no significant difference in academic cheating among Female adolescents having High Socio Economic Status, Middle Socio Economic Status and Low Socio Economic. 
4) There is no significant difference in academic cheating among Urban adolescents having High Socio Economic Status, Middle Socio Economic Status and Low Socio Economic.

5) There is no significant difference in Academic Cheating among Rural adolescents having High Socio Economic Status, Middle Socio Economic Status and Low Socio Economic.

\section{METHOD OF STUDY}

The study was carried out to investigate academic cheating among adolescents in relation to their socio-economic status. Accordingly descriptive survey method of research was used to conduct the study.

\section{Sample}

The sample comprised of 300 adolescents studying in different secondary schools of five districts i.e. Rohtak, Sonipat, Gurgaon, Fatheabaad and Yamunanagar district of Haryana State. A random sample of 150 adolescents (75 male and 75 female) from rural schools and 150 adolescents (75 male and 75 female) from urban schools formed the sample of the study.

\section{Tools used:-}

A self reported academic cheating Scale by Kalia and Kirandeep (2011) and Socio-economic status scale by Kalia and Sahu (2012) were used to assess academic cheating and socio-economic status of adolescents.

\section{Data Collection and Scoring:-}

Self reported academic cheating and socio-economic status questionnaires were administered to adolescents in their classroom settings. Before administering the test, the objectives of the study were explained to them. They were requested to respond each item honestly. On completion, the questionnaires were collected and scored as per directions given in the manual.

\section{Analysis of data}

One way ANNOVA was applied to test the significance of difference among different groups under consideration. In order to compare different groups ' $t$ ' test was applied.

Table No. 1(a) Means, SDs, \& SEMs of High Socio Economic Status, Middle Socio Economic Status and Low Socio Economic Status adolescents on Academic Cheating

\begin{tabular}{|c|c|c|c|c|c|}
\hline \multirow{2}{*}{$\begin{array}{c}\text { Sr. } \\
\text { No. }\end{array}$} & \multirow{2}{*}{$\begin{array}{c}\text { Socio Economic } \\
\text { Status }\end{array}$} & \multicolumn{3}{|c|}{ Academic Cheating } \\
\cline { 3 - 6 } I & $\begin{array}{c}\text { High Socio } \\
\text { Economic Status } \\
\text { adolescents }\end{array}$ & $\begin{array}{c}\text { Group 1 } \\
\text { No.118 }\end{array}$ & 57.84 & 32.09 & 2.95 \\
\hline II & $\begin{array}{c}\text { Middle Socio } \\
\text { Economic Status } \\
\text { adolescents }\end{array}$ & $\begin{array}{c}\text { Group 2 } \\
\text { No.90 }\end{array}$ & 51.66 & 28.67 & 2.98 \\
\hline III & $\begin{array}{c}\text { Low Socio } \\
\text { Economic Status } \\
\text { adolescents }\end{array}$ & $\begin{array}{c}\text { Group 3 } \\
\text { No.92 }\end{array}$ & 45.87 & 21.27 & 2.24 \\
\hline
\end{tabular}


Table No. 1 (b) Variance in the Academic Cheating among Adolescents having High Socio Economic Status, Middle Socio Economic Status and Low Socio Economic Status

\begin{tabular}{|c|c|c|c|c|}
\hline $\begin{array}{c}\text { Source of } \\
\text { Variation }\end{array}$ & $\boldsymbol{S S}$ & $\boldsymbol{d} \boldsymbol{f}$ & $\boldsymbol{M S}$ & $\boldsymbol{F}$ \\
\cline { 1 - 3 } $\begin{array}{c}\text { Between } \\
\text { Groups }\end{array}$ & 7381.65 & 2 & 3690.82 & 4.65 \\
\cline { 1 - 3 } $\begin{array}{c}\text { Within } \\
\text { Groups }\end{array}$ & 235638.89 & 297 & 793.39 & $\begin{array}{c}\text { 0.01 Level } \\
\text { of Sig. }\end{array}$ \\
\cline { 1 - 3 } Total & 243020.55 & 299 & & \\
\hline
\end{tabular}

From the table no. 1 (b) results of ANOVA indicate that F-ratio for the different groups came out to be 4.65 , which is statistically significant at 0.01 level of significance. It indicates that there is a significant difference among different groups. Different groups were compared using ' $t$ ' test. Table no. 1(c) depicts ' $t$ ' ratio among different groups.

Table No. 1(c) 't' ratios of High Socio Economic Status, Middle Socio Economic Status and Low Socio Economic Status adolescents on Academic Cheating

\begin{tabular}{|c|c|c|c|c|c|c|}
\hline \multirow{3}{*}{$\begin{array}{c}\text { Academic } \\
\text { Cheating }\end{array}$} & \multicolumn{2}{|c|}{ Group 1 \& Group 2} & \multicolumn{2}{|c|}{ Group 1 \& Group 3} & \multicolumn{2}{|c|}{ Group 2 \& Group 3} \\
\hline & 't' ratio & $\begin{array}{l}\text { Level } \\
\text { of sig. }\end{array}$ & $\begin{array}{l}\text { ' } \mathbf{t} \text { ' } \\
\text { ratio }\end{array}$ & $\begin{array}{c}\text { Level of } \\
\text { sig. }\end{array}$ & $\begin{array}{c}{ }^{6} t \\
\text { ratio }\end{array}$ & $\begin{array}{l}\text { Level of } \\
\text { sig. }\end{array}$ \\
\hline & 1.45 & N.S & 3.06 & 0.01 & 1.55 & N.S \\
\hline
\end{tabular}

From table no. 1(a) the Mean scores of different groups depicts that Group-1 i.e. High Socio Economic Status adolescents $(57.84 \pm 32.09)$ is highest in comparison with Middle Socio Economic Status adolescents (51.66 \pm 28.67) and Low Socio Economic Status adolescents $(45.87 \pm 21.27)$ ' $t$ ' ratio being 1.45 is statistically insignificant. It indicates that no significant difference was observed among Very High Socio Economic Status adolescents in comparison with Middle Socio Economic Status adolescents. However ' $t$ ' ratio being 3.06 found to be statistically significant at 0.01 level of significance, indicates that High Socio Economic Status adolescents were found to be significantly higher on academic cheating in comparison with Low Socio Economic Status adolescents.

The mean scores of Group-2 i.e. Middle Socio Economic Status (51.66 \pm 28.67$)$ is highest in comparison with Low Socio Economic Status adolescents (45.87 \pm 21.27 ), ' $t$ ' ratio being 1.55. it indicates that no significant difference was observed among Middle Socio Economic Status and Low Socio Economic Status adolescents on academic Cheating. 
Thus the hypothesis "There is no significant difference in academic cheating among adolescents having High Socio Economic Status, Middle Socio Economic Status and Low Socio Economic" is partially rejected.

Table No. 2(a) Means, SDs, \& SEMs of Male High Socio Economic Status, Male Middle Socio Economic Status and Male Low Socio Economic Status adolescents on Academic Cheating

\begin{tabular}{|c|c|c|c|c|c|}
\hline \multirow{2}{*}{$\begin{array}{l}\text { Sr. } \\
\text { No. }\end{array}$} & \multirow{2}{*}{$\begin{array}{c}\text { Socio Economic } \\
\text { Status }\end{array}$} & \multicolumn{4}{|c|}{ Academic Cheating } \\
\hline & & Groups & Mean & SD & SEM \\
\hline $\mathbf{I}$ & $\begin{array}{c}\text { Male High Socio } \\
\text { Economic Status } \\
\text { adolescents }\end{array}$ & $\begin{array}{c}\text { Group } 1 \\
\text { No.59 }\end{array}$ & 59.66 & 34.26 & 4.46 \\
\hline II & $\begin{array}{c}\text { Male Middle } \\
\text { Socio Economic } \\
\text { Status } \\
\text { adolescents }\end{array}$ & $\begin{array}{c}\text { Group } 2 \\
\text { No.45 }\end{array}$ & 50.11 & 28.55 & 4.25 \\
\hline III & $\begin{array}{c}\text { Male Low Socio } \\
\text { Economic Status } \\
\text { adolescents }\end{array}$ & $\begin{array}{c}\text { Group } 3 \\
\text { No.46 }\end{array}$ & 45.19 & 23.64 & 3.48 \\
\hline
\end{tabular}

Table No. 2(b) Variance in the Academic Cheating among Male Adolescents having High Socio Economic Status, Middle Socio Economic Status and Low Socio Economic Status

\begin{tabular}{|c|c|c|c|c|}
\hline $\begin{array}{l}\text { Source of } \\
\text { Variation }\end{array}$ & $S S$ & $d f$ & $M S$ & $F$ \\
\hline $\begin{array}{c}\text { Between } \\
\text { Groups }\end{array}$ & 5733.66 & 2 & 2866.82 & 3.26 \\
\hline $\begin{array}{l}\text { Within } \\
\text { Groups }\end{array}$ & 129122.9 & 147 & 878.38 & \multirow{2}{*}{$\begin{array}{l}\text { 0.05 Level } \\
\text { of Sig. }\end{array}$} \\
\hline Total & 134856.56 & 149 & & \\
\hline
\end{tabular}

From the table no.2 (b) results of ANOVA indicate that F-ratio for the different groups came out to be 3.26 , which is statistically significant at 0.05 level of significance. It indicates that different groups are statistically different from each other. Different groups were compared using ' $t$ ' test. Table no. 2(c) depicts ' $t$ ' ratio among different groups. 
Table No. 2(c) ' $t$ ' ratios of Male High Socio Economic Status, Male Middle Socio Economic Status and Male Low Socio Economic Status adolescents on Academic Cheating

\begin{tabular}{|c|c|r|c|c|c|c|}
\hline \multirow{2}{*}{$\begin{array}{c}\text { Academic } \\
\text { Cheating }\end{array}$} & \multicolumn{2}{|c|}{ Group 1 \& Group 2 } & \multicolumn{2}{|c|}{ Group 1 \& Group 3 } & \multicolumn{2}{c|}{ Group 2 \& Group 3 } \\
\cline { 2 - 7 } & 't' ratio & $\begin{array}{r}\text { Level } \\
\text { of sig. }\end{array}$ & $\begin{array}{c}\text { 't' } \\
\text { ratio }\end{array}$ & $\begin{array}{c}\text { Level of } \\
\text { sig. }\end{array}$ & $\begin{array}{c}\text { 't' } \\
\text { ratio }\end{array}$ & $\begin{array}{c}\text { Level of } \\
\text { sig. }\end{array}$ \\
\cline { 2 - 7 } & 1.51 & N.S & 2.44 & 0.05 & $\mathbf{0 . 8 9}$ & N.S \\
\hline
\end{tabular}

From table no. 2(a) the Mean scores of different groups depicts that Group-1 i.e. Male High Socio Economic Status adolescents $(59.66 \pm 34.26)$ is highest in comparison with Male Middle Socio Economic Status adolescents $(50.11 \pm 28.55)$ and Male Low Socio Economic Status adolescents $(45.19 \pm 23.64)$ ' $\mathrm{t}$ ' ratio being 1.51 is statistically insignificant. It indicates that no significant difference was observed among Male Very High Socio Economic Status adolescents in comparison with Male Middle Socio Economic Status adolescents. However ' $t$ ' ratio being 2.44 found to be statistically significant at 0.01 level of significance, indicates that Male High Socio Economic Status adolescents were found to be significantly higher on academic cheating in comparison with Male Low Socio Economic Status adolescents.

The mean scores of Group-2 i.e. Male Middle Socio Economic Status $(50.11 \pm 28.55)$ is highest in comparison with Low Socio Economic Status adolescents (45.19 \pm 23.64$)$, 't' ratio being 0.89 indicates that no significant difference was observed among Male Middle Socio Economic Status and Male Low Socio Economic Status adolescents on academic Cheating.

Thus the hypothesis that "There is no significant difference in academic cheating among Male adolescents having High Socio Economic Status, Middle Socio Economic Status and Low Socio Economic" is partially rejected.

Table No. 3(a) Means, SDs, \& SEMs of Female High Socio Economic Status, Female Middle Socio Economic Status and Female Low Socio Economic Status adolescents on Academic Cheating

\begin{tabular}{|c|c|c|c|c|c|}
\hline Sr. & Socio Economic & \multicolumn{4}{|c|}{ Academic Cheating } \\
\cline { 3 - 6 } No. & Status & Groups & Mean & SD & SEM \\
\hline I & $\begin{array}{c}\text { Female High Socio } \\
\text { Economic Status } \\
\text { adolescents }\end{array}$ & $\begin{array}{c}\text { Group 1 } \\
\text { No.59 }\end{array}$ & 56.02 & 29.96 & 3.9 \\
\hline II & $\begin{array}{c}\text { Female Middle } \\
\text { Socio Economic } \\
\text { Status adolescents }\end{array}$ & $\begin{array}{c}\text { Group 2 } \\
\text { No.47 }\end{array}$ & 53.14 & 29.02 & 4.23 \\
\hline III & $\begin{array}{c}\text { Female Low Socio } \\
\text { Economic Status } \\
\text { adolescents }\end{array}$ & $\begin{array}{c}\text { Group 3 } \\
\text { No.44 }\end{array}$ & 46.56 & 18.73 & 2.82 \\
\hline
\end{tabular}


Table No. 3(b) Variance in the Academic Cheating among Female Adolescents having High Socio Economic Status, Middle Socio Economic Status and Low Socio Economic Status

\begin{tabular}{|c|c|c|c|c|}
\hline $\begin{array}{c}\text { Source of } \\
\text { Variation }\end{array}$ & $\boldsymbol{S S}$ & $\boldsymbol{d f}$ & $\boldsymbol{M S}$ & $\boldsymbol{F}$ \\
\hline $\begin{array}{c}\text { Between } \\
\text { Groups }\end{array}$ & 2294.24 & 2 & 1147.12 & 1.59 \\
\hline Within Groups & 105869.74 & 147 & 720.2 & \multirow{2}{*}{ N.S } \\
\hline Total & 108163.97 & 149 & & \\
\hline
\end{tabular}

From the table no. 3 (b) results of ANOVA indicate that F-ratio for the different groups came out to be 1.59 , which is statistically insignificant. It indicates that no significant difference was observed among different groups. Different groups were compared using ' $t$ ' test. Table no. 3(c) depicts ' $t$ ' ratio among different groups.

Table No. 3(c) ' $t$ ' ratios of Female High Socio Economic Status, Female Middle Socio Economic Status and Female Low Socio Economic Status adolescents on Academic Cheating

\begin{tabular}{|c|c|c|c|c|c|c|}
\hline \multirow{2}{*}{$\begin{array}{c}\text { Academic } \\
\text { Cheating }\end{array}$} & \multicolumn{2}{|c|}{ Group 1 \& Group 2 } & \multicolumn{2}{|c|}{ Group 1 \& Group 3 } & \multicolumn{2}{|c|}{ Group 2 \& Group 3} \\
\cline { 2 - 7 } & 't' ratio & $\begin{array}{r}\text { Level } \\
\text { of sig. }\end{array}$ & $\begin{array}{c}\text { ' } t \text { ' } \\
\text { ratio }\end{array}$ & $\begin{array}{c}\text { Level of } \\
\text { sig. }\end{array}$ & $\begin{array}{c}\text { 't' } \\
\text { ratio }\end{array}$ & Level of sig. \\
\cline { 2 - 7 } & 0.49 & N.S & 1.84 & N.S & 1.28 & N.S \\
\hline
\end{tabular}

From table no. 3(a) the Mean scores of different groups depicts that Group-1 i.e. Female High Socio Economic Status adolescents $(56.02 \pm 29.96)$ is highest in comparison with Female Middle Socio Economic Status adolescents $(53.14 \pm 29.02)$ and Female Low Socio Economic Status adolescents $(46.56 \pm 18.73)$ ' $t$ ' ratio being 0.49 and 1.84 is statistically insignificant. It indicates that no significant difference was observed among Female High Socio Economic Status adolescents in comparison with Female Middle Socio Economic Status adolescents and Female Low Socio Economic Status adolescents.

The mean scores of Group-2 i.e. Female Middle Socio Economic Status (53.14 \pm 29.02$)$ is highest in comparison with Low Socio Economic Status adolescents (46.56 \pm 18.73$)$, ' $t$ ' ratio being 1.28 indicates that no significant difference was observed among Female Middle Socio Economic Status and Female Low Socio Economic Status adolescents on academic Cheating. 
Thus the hypothesis that "There is no significant difference in academic cheating among Female adolescents having High Socio Economic Status, Middle Socio Economic Status and Low Socio Economic" is accepted.

Table No. 4(a) Means, SDs, \& SEMs of Urban High Socio Economic Status, Urban Middle Socio Economic Status and Urban Low Socio Economic Status adolescents on Academic Cheating

\begin{tabular}{|c|c|c|c|c|c|}
\hline \multirow{2}{*}{$\begin{array}{l}\text { Sr. } \\
\text { No. }\end{array}$} & \multirow{2}{*}{$\begin{array}{c}\text { Socio Economic } \\
\text { Status }\end{array}$} & \multicolumn{4}{|c|}{ Academic Cheating } \\
\hline & & Groups & Mean & SD & SEM \\
\hline $\mathbf{I}$ & $\begin{array}{c}\text { Urban High Socio } \\
\text { Economic Status } \\
\text { adolescents }\end{array}$ & $\begin{array}{c}\text { Group } 1 \\
\text { No.36 }\end{array}$ & 61.14 & 28.99 & 4.83 \\
\hline II & $\begin{array}{c}\text { Urban Middle } \\
\text { Socio Economic } \\
\text { Status } \\
\text { adolescents }\end{array}$ & $\begin{array}{c}\text { Group } 2 \\
\text { No.53 }\end{array}$ & 53.77 & 27.14 & 3.72 \\
\hline III & $\begin{array}{c}\text { Urban Low Socio } \\
\text { Economic Status } \\
\text { adolescents }\end{array}$ & $\begin{array}{c}\text { Group } 3 \\
\text { No.61 }\end{array}$ & 49.07 & 21.22 & 2.72 \\
\hline
\end{tabular}

Table No. 4(b) Variance in the Academic Cheating among Urban Adolescents having High Socio Economic Status, Middle Socio Economic Status and Low Socio Economic Status

\begin{tabular}{|c|c|c|c|c|}
\hline $\begin{array}{c}\text { Source of } \\
\text { Variation }\end{array}$ & $\boldsymbol{S S}$ & $\boldsymbol{d f}$ & $\boldsymbol{M S}$ & $\boldsymbol{F}$ \\
\hline $\begin{array}{c}\text { Between } \\
\text { Groups }\end{array}$ & 3301.78 & 2 & 1650.88 & 2.56 \\
\hline $\begin{array}{c}\text { Within } \\
\text { Groups }\end{array}$ & 94759.33 & 147 & 644.62 & N.S \\
\hline Total & 98061.09 & 149 & & \\
\hline
\end{tabular}

From the table no. 4 (b) results of ANOVA indicate that F-ratio for the different groups came out to be 2.56, which is statistically insignificant. It indicates that no significant difference was observed among different groups. Different groups were compared using ' $t$ ' test. Table no. 4(c) depicts ' $t$ ' ratio among different groups. 
Table No. 4(c) 't' ratios of Urban High Socio Economic Status, Urban Middle Socio Economic Status and Urban Low Socio Economic Status adolescents on Academic Cheating

\begin{tabular}{|c|c|c|c|c|c|c|}
\hline \multirow{2}{*}{$\begin{array}{c}\text { Academic } \\
\text { Cheating }\end{array}$} & \multicolumn{2}{|c|}{ Group 1 \& Group 2 } & \multicolumn{2}{c|}{ Group 1 \& Group 3 } & \multicolumn{2}{c|}{ Group 2 \& Group 3 } \\
\cline { 2 - 7 } & 't' ratio & $\begin{array}{c}\text { Level } \\
\text { of sig. }\end{array}$ & $\begin{array}{c}\text { 't' } \\
\text { ratio }\end{array}$ & $\begin{array}{c}\text { Level of } \\
\text { sig. }\end{array}$ & $\begin{array}{c}\text { 't' } \\
\text { ratio }\end{array}$ & $\begin{array}{c}\text { Level of } \\
\text { sig. }\end{array}$ \\
\cline { 2 - 7 } & 1.22 & N.S & 2.36 & $\mathbf{0 . 0 5}$ & 1.03 & N.S \\
\hline
\end{tabular}

From table no. 4(a) the Mean scores of different groups depicts that Group-1 i.e. Urban High Socio Economic Status adolescents $(61.14 \pm 28.99)$ is highest in comparison with Urban Middle Socio Economic Status adolescents $(53.77 \pm 27.14)$ and Urban Low Socio Economic Status adolescents $(49.07 \pm 21.22)$ ' $t$ ' ratio being 1.22 is statistically insignificant. It indicates that no significant difference was observed among Urban High Socio Economic Status adolescents in comparison with Urban Middle Socio Economic Status adolescents. However ' $t$ ' ratio being 2.36 found to be statistically significant at 0.05 level of significance, indicates that Urban High Socio Economic Status adolescents were found to be significantly higher on academic cheating in comparison with Urban Low Socio Economic Status adolescents.

The mean scores of Group-2 i.e. Urban Middle Socio Economic Status (53.77 \pm 27.14$)$ is highest in comparison with Low Socio Economic Status adolescents (49.07 \pm 21.22 ), 't' ratio being 1.03 indicates that no significant difference was observed among Urban Middle Socio Economic Status and Urban Low Socio Economic Status adolescents on academic Cheating.

Thus the hypothesis that "There is no significant difference in academic cheating among Urban adolescents having High Socio Economic Status, Middle Socio Economic Status and Low Socio Economic" is partially accepted.

Table No. 5(a) Means, SDs, \& SEMs of Rural High Socio Economic Status, Rural Middle Socio Economic Status and Rural Low Socio Economic Status adolescents on Academic Cheating

\begin{tabular}{|c|c|c|c|c|c|}
\hline \multirow{2}{*}{$\begin{array}{l}\text { Sr. } \\
\text { No. }\end{array}$} & \multirow{2}{*}{$\begin{array}{c}\text { Socio Economic } \\
\text { Status } \\
\end{array}$} & \multicolumn{4}{|c|}{ Academic Cheating } \\
\hline & & Groups & Mean & SD & SEM \\
\hline I & $\begin{array}{c}\text { Rural High Socio } \\
\text { Economic Status } \\
\text { adolescents }\end{array}$ & $\begin{array}{c}\text { Group } 1 \\
\text { No.82 }\end{array}$ & 56.39 & 33.44 & 3.69 \\
\hline II & $\begin{array}{c}\text { Rural Middle } \\
\text { Socio Economic } \\
\text { Status } \\
\text { adolescents }\end{array}$ & $\begin{array}{c}\text { Group } 2 \\
\text { No.39 }\end{array}$ & 48.79 & 30.75 & 4.92 \\
\hline III & $\begin{array}{c}\text { Rural Low Socio } \\
\text { Economic Status } \\
\text { adolescents }\end{array}$ & $\begin{array}{c}\text { Group } 3 \\
\text { No.29 }\end{array}$ & 39.13 & 20.12 & 3.73 \\
\hline
\end{tabular}


Table No. 5(b) Variance in the Academic Cheating among Rural Adolescents having High Socio Economic Status, Middle Socio Economic Status and Low Socio Economic Status

\begin{tabular}{|c|c|c|c|c|}
\hline $\begin{array}{c}\text { Source of } \\
\text { Variation }\end{array}$ & $\boldsymbol{S S}$ & $\boldsymbol{d} \boldsymbol{f}$ & $\boldsymbol{M S}$ & $\boldsymbol{F}$ \\
\cline { 1 - 3 } $\begin{array}{c}\text { Between } \\
\text { Groups }\end{array}$ & 6651.72 & 2 & 3325.86 & 3.54 \\
\cline { 1 - 3 } $\begin{array}{c}\text { Within } \\
\text { Groups }\end{array}$ & 137821.32 & 147 & 937 & \multirow{2}{*}{$\mathbf{0 . 0 5}$} \\
\cline { 1 - 3 } Total & 144473.04 & 149 & & \\
\hline
\end{tabular}

From the table no. 5 (b) results of ANOVA indicate that F-ratio for the different groups came out to be 3.54 , which is statistically significant at 0.05 level of significance. It indicates that significant difference was observed among different groups. Different groups were compared using ' $\mathrm{t}$ ' test. Table no. 5(c) depicts ' $\mathrm{t}$ ' ratio among different groups.

Table No. 5(c) 't' ratios of Rural High Socio Economic Status, Rural Middle Socio Economic Status and Rural Low Socio Economic Status adolescents on Academic Cheating

\begin{tabular}{|c|c|c|c|c|c|c|}
\hline & \multicolumn{2}{|c|}{ Group 1 \& Group 2 } & \multicolumn{2}{c|}{ Group 1 \& Group 3} & \multicolumn{2}{c|}{ Group 2 \& Group 3} \\
\cline { 2 - 7 } $\begin{array}{c}\text { Academic } \\
\text { Cheating }\end{array}$ & 't' ratio & $\begin{array}{c}\text { Level } \\
\text { of sig. }\end{array}$ & $\begin{array}{c}\text { ' } t \text { ' } \\
\text { ratio }\end{array}$ & $\begin{array}{c}\text { Level of } \\
\text { sig. }\end{array}$ & $\begin{array}{c}\text { 't' } \\
\text { ratio }\end{array}$ & $\begin{array}{c}\text { Level of } \\
\text { sig. }\end{array}$ \\
\cline { 2 - 7 } & 1.19 & N.S & 2.61 & 0.01 & 1.47 & N.S \\
\hline
\end{tabular}

From table no. 5(a) the Mean scores of different groups depicts that Group-1 i.e. Rural High Socio Economic Status adolescents (56.39.14 \pm 33.44) is highest in comparison with Rural Middle Socio Economic Status adolescents (48.79 \pm 30.75) and Rural Low Socio Economic Status adolescents $(39.13 \pm 20.12)$ ' $t$ ' ratio being 1.19 is statistically insignificant. It indicates that no significant difference was observed among Rural High Socio Economic Status adolescents in comparison with Rural Middle Socio Economic Status adolescents. However ' $t$ ' ratio being 2.61 found to be statistically significant at 0.01 level of significance, indicates that Rural High Socio Economic Status adolescents were found to be significantly higher on academic cheating in comparison with Rural Low Socio Economic Status adolescents.

The mean scores of Group-2 i.e. Rural Middle Socio Economic Status (48.79 \pm 30.75$)$ is highest in comparison with Low Socio Economic Status adolescents (39.13 \pm 20.12$)$, 't' ratio being 1.47 indicates that no significant difference was observed among Rural Middle Socio Economic Status and Rural Low Socio Economic Status adolescents on academic Cheating. 


\section{Academic Cheating among Adolescents in relation to Socio-Economic Status}

Thus the hypothesis that "There is no significant difference in academic cheating among Rural adolescents having High Socio Economic Status, Middle Socio Economic Status and Low Socio Economic" is partially rejected.

\section{SUMMARY AND CONCLUSIONS}

Academic Cheating among high socio-economic status adolescents were found significantly higher in comparison with low socio-economic status adolescents. Similar results were observed in case of Male, Urban and Rural adolescents for same groups' comparison.

$>$ There is no significant difference in academic cheating among female adolescents having high socio-economic status, middle socio-economic status and low socio-economic status.

$>$ No significant difference was found among middle socio-economic status and low socioeconomic status adolescents. Results were alike for Male, Female, Urban and Rural adolescents for same groups' comparison.

$>$ Also academic cheating among high socio-economic status and middle socio-economic status adolescents was found to be on same level for Male, Female, Urban and Rural adolescents.

\section{REFERENCES}

Aduloju, M. O. and Obinne, E. A. D. (2013). Assessment of Sex and Parental Socio-Economic Factors in Examination Cheating Behavior among University Students: Implication for Measurement of Intellectual Functioning and Adjustment. Open Journal of Education, $1(7), 177-181$.

Alam, M. M. (2009). "Effect of Creativity and Socio-Economic Status of Students on Academic Achievement", Indian Psychological Review, 72(1), 35-39.

Kalia, A. K. \& Dalal, K. (2011). Manual of Academic Cheating Scale, Agra. National Psychological Corporation.

Kalia, A.K \& Sahu (2012). Manual of Socio Economic Status Scale, Agra. National Psychological Corporation.

Khan. I., \& Khan. M.J. (2011). Socio-economic status of students and malpractices used in Examinations in urban areas of district Peshawar. European Journal of scientific research, 49(4), 601-609.

McCabe, D.L. (1999). Academic dishonesty among high school students. Journal of Adolescence, 34, 681-687.

McCabe, D.L., Trevino, L.K. \& Butterfield, D.K. (2001). Cheating in academic Institutions: A decade of research. Ethics and Behaviour, 11(3), 219-232.

Murdock, T. B., Hale, N. M., \& Weber, M. J. (2001). Predictors of cheating among early adolescents: Academic and social motivations. Contemporary Educational Psychology, 26, 96-115. 


\section{Academic Cheating among Adolescents in relation to Socio-Economic Status}

Okorodudu, G. N. (2013). Peer Pressure and Socioeconomic Status as Predictors of Student's Attitude to Examination Malpractice in Nigeria. International Journal of Education, 5(1), 36-51.

Pandey, S. K. and Maikhuri, R. (2003). "Socio-Economic Status and Academic Achievement", Journal in PsychoLingua, 33 (1), 60-64.

Panigrahi, M.R. (2005). "Academic Achievement in Relation to Intelligence and SocioEconomic Status of High School Students", Edutracks, 5(2), 26-27.

Stephens, J. M. and Nicholson, H. (2008). Cases of incongruity: exploring the divide between adolescents' beliefs and behavior related to academic dishonesty. Educational Studies. 34(4), 361-376. 